LAWRENCE LIVERMORE N A T IO N A L LABORATORY
Double-shell target design for the NIF: Noncryogenic ignition and nonlinear mix studies for Stockpile Stewardship

Peter Amendt

February 17, 2004 
This document was prepared as an account of work sponsored by an agency of the United States Government. Neither the United States Government nor the University of California nor any of their employees, makes any warranty, express or implied, or assumes any legal liability or responsibility for the accuracy, completeness, or usefulness of any information, apparatus, product, or process disclosed, or represents that its use would not infringe privately owned rights. Reference herein to any specific commercial product, process, or service by trade name, trademark, manufacturer, or otherwise, does not necessarily constitute or imply its endorsement, recommendation, or favoring by the United States Government or the University of California. The views and opinions of authors expressed herein do not necessarily state or reflect those of the United States Government or the University of California, and shall not be used for advertising or product endorsement purposes.

This work was performed under the auspices of the U.S. Department of Energy by University of California, Lawrence Livermore National Laboratory under Contract W-7405-Eng-48. 


\title{
Double-shell target design for the NIF: Noncryogenic ignition and nonlinear mix studies for stockpile stewardship
}

\author{
Peter Amendt, PI \\ 01-ERD-033 Final Report \\ LLNL, Livermore, California
}

\begin{abstract}
Double-shell ignition is complementary to the baseline approach by virtue of not requiring: (1) cryogenic preparation and fielding, (2) high-contrast pulse-shaping for shock-timing, and (3) demanding x-ray flux symmetry control. The use of simpler lowcontrast pulse-shaping potentially allows more benign hohlraum conditions by reducing the risk of laser backscatter. In addition, the associated higher laser fluence threshold for optics damage initiation allows the possibility of more routine high-fluence shots with $2 \omega$ on the NIF.

Based on LDRD-sponsored research in FY01-03 on NIF double-shell ignition target designs, the feasibility of this approach was advanced through both a highly successful implosion campaign on the Omega laser facility and a variety of design improvements for mitigating instability. The double-shell implosion campaign on Omega achieved the important milestone of repeatably demonstrating dominant primary (2.45 $\mathrm{MeV}$ ) neutron production from the mix-susceptible compressional phase of a double-shell implosion, using fall-line design optimization and exacting fabrication standards. Showing effective control of fuel-pusher mix during final compression is an essential element for achieving ignition. In our studies to control mix by reducing hydrodynamic instability a new pathway for destructive Rayleigh-Taylor growth on the outer surface of the inner shell at ignition scales was identified. However, highly resolved multi-mode simulations showed that with use of a graded dopant in the inner shell and materialmatching with an exterior metallic foam, this instability was significantly reduced. In addition, the resulting density-gradient stabilization was seen to quench small-wavelength growth, thereby avoiding the computationally challenging turbulent regime. A major goal of future research for realizing double-shell ignition on the NIF is experimental validation of this instability mitigation approach using the Omega laser facility.
\end{abstract}

\section{Introduction and motivation}

Lawrence Livermore National Laboratory is committed to achieving ignition as soon as possible on the NIF. To this end, the inertial confinement fusion effort is focussed on cryogenic singleshell implosion designs because of their potential for high gain. An additional ignition option is the double shell. This design [See Fig. 1], in spite of producing lower thermonuclear yields (3$7 \mathrm{MJ}$ ), does have some significant advantages. First, the double shell ignition capsule is not cryogenic, holding its DT fuel at room temperature. Second, the necessary peak implosion velocity is nearly a factor-of-two lower, providing for reduced in-flight shell aspect ratio and higher margin to instability feed-through in the inner shell. Third, symmetry tuning is easier for the double shell design, due to the modest three-fold convergence (or initial radius over final radius) of the outer shell and ten-fold convergence of the inner shell. Fourth, the ignition temperature is lower $(\approx 4 \mathrm{keV})$ compared to cryogenic single shell design $(\approx 10 \mathrm{keV})$, enabling yet more margin to capsule and hohlraum radiation asymmetries. Fifth, the laser power history demands are more relaxed than in the single shell design, requiring very little pulse shaping. Sixth, the double shell ignition design requests high laser power only at early time, thereby 
allaying concerns about hohlraum filling and the associated potential for plasma-mediated laser backscatter.

To appreciably increase our confidence in achieving ignition on the NIF, pursuit of a complementary approach to the baseline single-shell ignition design is prudent. The noncryogenic double-shell target represents such a complementary approach [2]. Besides enjoying the advantages enumerated above, it is complementary in the following additional respects. First, the in situ, dense, high- $Z$ inner shell acts as a pusher for the interior DT fuel, using spherical convergence and compressibility to reach the necessary high density for inertial confinement. Thus, there is little need for careful shock-sequencing to pre-form the pusher from lower density DT ice as in the cryogenic baseline design. Second, the high- $Z$ nature of the inner shell traps the radiation emanating from the hot fuel, leading to more LTE-like conditions of the burning fuel. The effect of radiative trapping leads to a lower threshold ignition temperature $(\approx 4 \mathrm{keV})$, compared with $\approx 10 \mathrm{keV}$ for the baseline design listed above. With less need for careful shock sequencing, a large degree of freedom is available for the laser power history. Instead of the 50:1 contrast pulse-shape envisioned for the baseline cryogenic design, a simpler and potentially more benign pulse-shape is accessible to double-shells. For example, a complementary "reverse-ramp" pulse-shape with requested peak power at early time is possible. The reverse-ramp laser pulse-shape means that at the instant of peak laser power, the hohlraum has had little time to fill with plasma. Such hohlraum conditions are benificial from the standpoint of reducing the potential for plasma-mediated laser backscatter and cross-beam energy transfer. Finally, the mode of ignition is intrinsically volume-like in the sense that the entire fuel burns nearly simultaneously as opposed to the propagating burn scenario with hot-spot ignition. Such a mode of ignition is interesting in its own rite, serving as a complementary test bed to the baseline target for studying ignition physics.

These advantages of a double-shell path to ignition lead to attendant design and fabrication challenges, which are also complementary in many respects to the baseline design. First, the noncryogenic double-shell target option is not high-gain, mainly because of the order-ofmagnitude less DT fuel mass. However, the burn fraction is higher and reaches nearly $70 \%$ according to simulation studies. Second, all four interfaces of the double-shell target are hydrodynamically unstable at one time or another during the implosion. Although the outer surface of the outer shell is ablatively stabilized for high mode numbers, the other three interfaces are classically unstable with relatively high Atwood numbers. The principal challenge with double-shell research has been to reliably model these interfaces and to explore design options for mitigating the instabilities to the point that the inner shell remains intact. Considerable progress has been made in this area [3] and is described further in the next section. Third, the high- $Z$ nature of the inner shell implies that nearly all hard $x$ rays generated in the hohlraum that reach the inner shell will be absorbed. This feature introduces another potential source of flux asymmetry, which must be remedied. For the baseline target design, there is far less sensitivity to x-ray preheat than with the double shell target. However, much progress has been made to accommodate and correct for this effect in integrated double-shell simulations [2]. Fourth, the fabrication of double-shell targets introduces a whole new set of challenges which in some respects are more stringent than for the baseline design. Some of these fabrication challenges include providing ultra-smooth surfaces for margin against hydrodynamic instability, ensuring shell concentricity to within 5 microns, and developing the capability to fill a seamless inner shell with greater than $700 \mathrm{~atm}$ of DT gas while safely containing this gas at room temperature. These are formidable fabrication requirements, requiring a comprehensive study of candidate double-shell materials and assembly methods, testing, and exacting characterization. Fifth, the dense high- $Z$ inner shell at peak compression stresses our diagnostic capability for imaging and characterizing the fuelpusher interface. The success of double-shell ignition will largely depend on the extent of instability-induced mixing of fuel and pusher material. A means of diagnosing this effect, 
through hard x-ray imaging (> $100 \mathrm{keV}$ ), radiochemistry, or neutron and charged particle imaging, will need to be developed and tested.

In summary, double-shell ignition is complementary in many respects to the baseline design. Thus, a concurrent effort on developing a comprehensive plan for double-shell ignition avoids duplication of the baseline effort and provides an independent path toward improving the overall prospects that ignition will be achieved on the NIF.

\section{Status of double-shell research}

Until a few years ago, the prospects for laser-driven double-shell ignition appeared quite tenuous. First, there was John Lindl's early assessment that the onset of turbulence after shell collision would inevitably lead to inner-shell breakup [4]. Second, the experience of Los Alamos researchers with ignition-like double-shell experiments on the Nova and Omega lasers gave consistently low performance. However, the situation began to progress with a successful set of experiments by LANL on Omega four years ago [5]. Although the targets were not ignition-like, these double shells performed with near 1-D behavior for the first time in a laser-driven experiment. Buoyed by this development and the availability of sophisticated simulation tools, a team of researchers at LLNL undertook a three year LDRD-sponsored investigation to assess the feasibility of double-shell ignition on the NIF. The effort was three-fold in nature, requiring analytic, computational and experimental resources. The main results and accomplishments of this study may be summarized as follows:

- Successfully performed two-dimensional integrated hohlraum simulation tunes of NIF double-shell targets driven at 200 and $250 \mathrm{eV}$.

- Introduced a "fall-line" design metric for minimizing the effect of mix in proposed ignition double-shell targets.

- Discovered the sensitivity of double-shells to hard x-ray flux asymmetry and developed an in situ diagnostic technique for testing on Omega.

- Designed NIF laser power histories with reduced damage initiation and plasma-mediated backscatter, thereby allowing more driver energy.

- Discovered principal mode of double-shell disruption from hydrodynamic instability using state-of-the-art computational tools and unprecedented numerical resolution.

- Devised a novel double-shell target design with graded dopant and foams for instability control, giving near 1-D behavior in highly-resolved 2-D simulations.

- Successfully completed Omega experimental campaign with ignition-like low-Z double-shell implosion behavior.

Some of these research highlights warrant additional discussion and emphasis. First, the firm identification of the instability mechanism responsible for inner-shell disruption provided the needed basis for a later corrective design [3]. This improved design exploited a graded dopant in the inner shell and an exterior metallic foam to provide an improved density match for suppressing dangerous (near classical) Rayleigh-Taylor instability growth. Highly resolved 2D simulations of an imploding double shell with surface roughness perturbations out to Legendre mode number $\approx 800$ show near 1 -D behavior, a dramatic improvement over recent designs using low- to mid-Z tampers for instability control. This development basically counters the 
longstanding perception that double-shell ignition targets are too plagued by instability growth to take seriously as an ignition option. Second, the recent double-shell implosion campaign on Omega was a testament to our understanding of double-shell implosion behavior and the ability to fabricate such targets to exacting ignition-like tolerances [6]. Prior to this campaign, the LANL double-shell effort was not able to repeatably demonstrate ignition-like behavior on existing laser systems, i.e., Nova and Omega. The high performing targets mentioned earlier [5] were unfortunately not ignition-like in the sense that a large fraction of the neutrons yields that were generated occurred near the instant of shock coalescence instead of the latter, ignition-relevant, stagnation phase. Current ignition designs require that the overriding majority of energy production occur near peak compression when the susceptibility to fuel-pusher mix is greatest. The significant feature of our Omega double-shell campaign is that we were able to repeatably demonstrate quite dominant neutron production near peak compression. In summary, an understanding of ignition-like double-shell behavior has been demonstrated in the laboratory for the first time.

\section{Future challenges in double-shell research}

As great as the progress in double-shell research has been over the past three years, significant hurdles remain in realizing ignition on the NIF. To comprehensively address these challenges, three areas of intensive investigation are foreseen: (1) Design optimization and robustness; (2) Experimentation on existing laser facilities; and (3) Target development, fabrication and characterization. Specifics on these three areas of research are detailed below.

\section{IIIa. Design optimization and robustness}

A host of design challenges and tasks remains in establishing confidence in double-shell ignition on NIF. These include: (1) two- and three-dimensional integrated hohlraum simulations for a variety of hohlraum configurations; (2) two- and three-dimensional capsuleonly highly resolved surface perturbation studies and target optimization; (3) parameter sensitivity studies to determine an optimal suite of ignition designs; (4) scoping studies for assessing the viability of directly-driven ignition double-shells in an indirect-drive laser configuration; (5) molecular dynamics simulation studies of atomic transport between the fuel and the high-Z pusher; and (6) analysis of relevant past experiments.

(1) Presently, integrated hohlraum symmetry tunes exist only for 200 and $250 \mathrm{eV}$ drive temperatures. These preliminary two-dimensional tunes were completed early in 2001 and were restricted to $3 \omega$ laser frequency. Further simulations studies are needed to explore additional hohlraum drive temperatures, pulse-shaping, $2 \omega$ laser drive, varying hohlraum compositions including gas-fill and mid-Z liners, and optimized beamphasing. The capability for three-dimensional integrated hohlraum simulations now exists and should be applied to the optimized two-dimensional tunes. In addition, recent work indicates that our mainline design code tends to considerably underestimate hard x-ray preheat at early time, to which the inner shell of a double-shell target is particularly sensitive. The impact of this experimentally confirmed effect on present and future double-shell ignition designs requires further assessment.

(2) Our stability investigations over the past year have concentrated on $\mathrm{Au} / \mathrm{Cu}$ inner shells and were restricted to two-dimensional simulations. Ultimately, three-dimensional, capsule-only simulations will be required to ensure robustness of our suite of designs to hydrodynamic instability. Other target materials need to be explored and assessed for compatibility with existing and projected fabrication and characterization methods. 
For example, design studies over the past year have identified $\mathrm{Pt}-\mathrm{Ir}, \mathrm{W}, \mathrm{Au}-\mathrm{Cu}, \mathrm{Ti}, \mathrm{Mo}$, and $\mathrm{Pd}$ as favorable candidate inner-shell materials. Other foam types and densities also need to be explored for optimum performance.

(3) Parameter variation and sensitivity studies of the integrated target designs are essential for deciding on an optimally robust target. In particular, sensitivity to beam mispointings, laser power imbalances, shell nonconcentricity, shell thickness variations, dopant inhomogeneities, target mispositioning, fabrication joints and seams, surface roughnesses, opacity and drive uncertainties, and foam porosity need to be understood and quantified.

(4) The LDRD-sponsored research on double-shell ignition target designs focussed exclusively on hohlraum-driven configurations. An option that has not been explored to date is directly-driven double-shell ignition. The University of Rochester over the past year has expended considerable effort in designing a cryogenic, single-shell capsule for fielding on the NIF in the indirect-driven laser configuration ("Polar Direct-Drive"). This effort has encountered difficulty in finding a satisfactory symmetry tune. However, the relatively low ignition temperature of a double-shell relaxes the symmetry requirements and may enable ignition with directly-driven double shells.

(5) Ultimately, the success or failure of an ignition double shell will likely rest on the degree of mixing between the low-Z DT fuel and the high- $Z$ pusher. Assessing the properties of such a mixing flow requires reliably understood transport properties - in particular, viscosity and mass diffusion. Both atomic transport processes are predicted to be important in reducing Rayleigh-Taylor and Richtmyer-Meshkov instability for high wavenumber surface perturbations [7]. Because of the large charge asymmetry of the mixing plasmas, phase separations may arise and appreciably affect transport. Molecular dynamics simulations can provide a powerful tool for exploring such aspects of atomic tranport from first principles.

(6) The existing double-shell database could provide further clues and information on potential vulnerabilities of existing designs. For example, recent LANL double-shell campaigns could shed light on the effect of large pore-size foams on destructive hydrodynamic instability.

Much of the design challenges just described would make use of simulation techniques and computational resources that simply did not exist ten years ago. A large part of the recent LDRD-sponsored progress on double-shell target design is attributable to the availability of such tools at LLNL. With expected advances in computer resources over the near term further improvements in design robustness and the ability to rapidly identify potential failure modes will be at hand.

\section{IIIb. Experiments on existing facilities}

The recent progress shown with double-shell implosions on Omega goes some distance in establishing confidence in our ability to model such targets. However, the energy limitations of the Omega laser restrict us to low- $Z$ inner shells for the most part. For mid- to high- $Z$ materials, the inner shells would have to be rather thin to allow a sufficient (or ignition-like) implosion velocity for a given driver energy. But such imploding thin shells are very susceptible to feed-through of hydrodynamic instability. Still, the resources of the Omega laser have hardly been exhausted and continued uses for double-shell research are foreseen. An expanded double-shell campaign on Omega would be instrumental in addressing three main 
challenges: (1) demonstrating continued robust double-shell performance beyond polystyrene $(\mathrm{CH})$ inner shells, (2) demonstrating control of instability growth as validation of our reduced instability ignition designs, and (3) optimizing surrogate diagnostic techniques for measuring $\mathrm{x}$ ray preheat strength and asymmetry near target center.

(1) Despite the energy limitations of the Omega laser, significant latitude remains in contemplating higher- $Z$ inner-shell materials. The logical and practical next material to consider is an all-glass $\left(\mathrm{SiO}_{2}\right)$ inner shell. However, this choice of inner shell has generally met with failure in the past. A number of hypotheses have been advanced, including Au M-band (2-4 keV) x-ray asymmetry, shell nonconcentricity, impurities in the glass inner shell, instability induced by large pore-size foams or an inner-shell web support, and outer shell imperfections such as opacity mismatched epoxy or gaps between the component hemispherical shells. As these targets were exclusively built and fielded by LANL researchers, we can't declare with any confidence which of these candidate effects could be dominant. The current challenge is to apply the fabrication and assembly techniques successfully demonstrated with the $\mathrm{CH}$ double shells towards an all-glass inner-shell version. Design criteria applied to the $\mathrm{CH}$ double-shell targets would also be exercised; namely, optimizing the fall-line behavior to reduce the effects of fuel-pusher mix. Another challenge with an all-glass inner shell is the increased radiative cooling effect of glass compared with $\mathrm{CH}$, giving more sensitivity to atomic mix $(\approx 3 x)$ and reduced implosion performance.

(2) Experimental validation of our graded-dopant double-shell design is essential for gaining confidence in an ignition plan. The Omega facility could provide a useful venue for testing instability-control with graded dopants. For example, double planar experiments with a sandwiched foam could be used as a testbed for studying control of Rayleigh-Taylor instability on the outer surface of the inner shell (or slab) over a variety of conditions. Robust experimental designs are needed to unambiguously detect and measure instability growth well into the nonlinear regime.

(3) Over the past two years an M-band diagnostic target has been developed and fielded on the Omega laser with promising results. The target consists of a double-shell configuration with an oversized outer shell for filtering out thermal $x$ rays originating from the hohlraum walls, allowingthe harder $x$ rays to reach a higher- $Z$ inner shell. The inner shell is made of glass, which is an efficient absorber of M-band $x$ rays, and a plastic tamper for reducing the outward expansion of the heated glass. The motion of the converging inner shell is monitored with backlighting to assess the M-band $\mathrm{x}$-ray fluence at target center. To date, useful data have been obtained on the average trajectory of the imploding inner shell, but only modest signal-to-noise levels have prevented any useful symmetry information. Further development of this technique is needed to extract low-order x-ray preheat asymmetry information. In addition, the limitations of the Omega laser have constrained us to consider only M-band x-ray effects (in $\mathrm{Au}$ hohlraums), whereas simulations of NIF double-shell ignition hohlraums indicate a large effect from L-shell $x$ rays $(>8 \mathrm{keV})$. L-shell effects could be studied on Omega but at the expense of fielding lower- $Z$ hohlraums which clearly are less NIF-relevant.

The above experimental challenges with double-shell research emphasize the Omega laser as a principal testbed. However, use of the NIF could help address some of these challenges as well, subject to the availability of shots. Although a symmetric laser configuration will not be available until FY09, planar experiments to study instability phenomenona and L-shell effects could be designed and fielded in the interim. 


\section{IIIc. Target fabrication, development and characterization}

A sustained and dedicated effort in fabrication, target development and characterization is key to realizing noncryogenic ignition. Fabrication of projected targets for experiments on Omega will involve application of established and demonstrated techniques for the most part. A concurrent path on developing the material science requirements, techniques for precision micro-assembly and nondestructive characterization of prototypical ignition targets is critically essential. In the area of material science development, several areas of intensive research are needed, including (1) an assessment of candidate inner-shell alloys in the areas of yield strength, hydrogen diffusivity, and surface finish properties; (2) developing minimally invasive bonding methods for joining the outer-shell hemispheres; (3) creating a suite of lowdensity, small cellsize, configurable, metallic foams; (4) accomodation of suitable dopant gradients in the inner shell; and (5) analysis and testing of techniques for high-pressure DT filling of the inner shell.

(1) In the past year a suite of inner-shell materials has been proposed for double-shell ignition on the NIF. The preponderance of analysis and simulations has been devoted to (86-14\%) Au-Cu alloys, but other candidate materials may be more advantageous from the standpoint of mix-mitigation and fabricability. In considering a candidate material, four basic elements must be addressed: (1) ability of the inner shell to safely contain greater than $700 \mathrm{~atm}$ DT at room temperature, (2) compatibility with diffusionfilling of DT, (3) surface roughness properties including granularity, anisotropy, and accessibility to various smoothing techniques, and (4) the ability to accommodate graded dopants.

(2) Current fabrication methods for Omega-scale double shells require the bonding of two hemispherical outer shells with epoxy. Simulation studies are needed to assess the affect of such a seamed outer shell on double-shell performance for the NIF. Methods for ensuring a minimally invasive joined seam must be developed.

(3) Design studies have suggested a distinct advantage for stability control in having an inter-shell foam material which compositionally matches the outside surface of the inner shell. With the suite of inner shell materials currently envisioned for the NIF a corresponding suite of low-density, small pore size, metallic foam materials requires research and development.

(4) In tandem with use of carefully chosen foams between the two shells, the use of graded dopants in the inner shell are likely required for instability mitigation. For example, simulation studies suggest that an inner region of $\mathrm{Au}$ in the inner shell followed by successively larger amounts of $\mathrm{Cu}$ towards the outer surface has desirable stability properties [3]. However, the grading profile must be tailored to provide sufficient yield strength in the inner shell while ensuring a suitably smooth transition to pure $\mathrm{Cu}$ at the outer surface of the inner shell.

(5) Methods for reliably and safely filling high-Z inner shells with $700 \mathrm{~atm}$ of DT gas are required. Several techniques have been proposed for this purpose, including (1) hightemperature diffusion filling, (2) diffusion filling via a decomposable palladium valve, and (3) focused ion beam technology. In the first approach, the shell is subjected to high temperature for enhancing hydrogen diffusivity and has the advantage of not 
leaving a structural feature on the inner shell. The second approach uses a palladium stalk through which DT can readily diffuse. Following the high-pressure filling, the stalk is heated which then readily diffuses into the inner shell. The third approach is a fill-and-plug method, which uses a focused ion beam to drill a hole with sub-micron bore size. All of these filling techniques represent challenging and untested approaches toward addressing a critical step in realizing ignition double shell targets, requiring considerable investment in research and development.

\section{References}

[1] S.W. Haan et al., Phys. Plasmas 2, 2480 (1995).

[2] P. Amendt et al., Phys. Plasmas 9(5), 2221 (2002).

[3] J.L. Milovich et al., Phys. Plasmas, to appear (2004).

[4] J.D. Lindl, Inertial Confinement Fusion (Springer-Verlag, New York, 1998).

[5] W.S. Varnum et al., Phys. Rev. Lett. 84, 5153 (2000).

[6] P. Amendt et al., Phys. Rev. Lett., submitted (2004).

[7] P. Amendt et al., Phys. Plasmas 10(3), 820 (2003).

\section{Figure captions}

Fig. 1: Schematic of NIF double-shell target design driven at $250 \mathrm{eV}$ hohlraum temperature. 